\title{
3D LIVE HUMANS IN MIXED REALITY ENTERTAINMENT
}

Simon J.D. Prince ${ }^{1}$, Adrian David Cheok ${ }^{1}$, Farzam Farbiz ${ }^{1}$, Todd Williamson ${ }^{2}$, Nik Johnson ${ }^{2}$, Mark Billinghurst ${ }^{3}$ and Hirokazu Kato ${ }^{4}$ National University of Singapore ${ }^{1}:$ Zaxel Systems $^{2}:$ University of Washington $^{3}:$ Hiroshima City University $^{4}$

Abstract: $\quad$ We present a system that introduces live captured 3-d content into augmented reality. For each frame we estimate the transformation between the users point of view and a card marker. We then generate a novel or "virtual" view of the live subject from the same perspective. This "virtual view" is generated based on measurements from fifteen cameras that surround the subject. A fast shape-from-silhouette algorithm reconstructs a 3-d model of the live subject from the user's perspective and re-samples the original images to generate the novel view. This is then rendered into the user's world at interactive speeds. The result gives the strong impression that the subject is a real part of the 3-D scene. We present a novel mixed reality entertainment application in which users apply tangible computing techniques to interact with a collaborator in a virtual space.

Key words: Mixed reality, 3-D reconstruction, Computer Entertainment, Computer Vision.

\section{INTRODUCTION}

A critical limitation of multi-player computer entertainment applications is the inability to effectively visualise other users. The current approach to this problem is to introduce a symbolic graphical representation (avatar) to represent each player. However, the uniqueness and expressiveness of such representations are inherently limited. Indeed, in the virtual environments literature, there has been considerable research into identifying those nonverbal behaviours that are crucial for collaboration [3] and elaborate

The original version of this chapter was revised: The copyright line was incorrect. This has been corrected. The Erratum to this chapter is available at DOI: 10.1007/978-0-387-35660-0_65 
interfaces have been developed to control expression in avatars. The aim of this paper is to solve these problems by replacing the symbolic avatar with a simulated view of the actual user, which is captured at interactive speeds.

In addition to introducing images of real users into purely graphical environments, this system can also be applied to develop augmented reality entertainment applications. Augmented reality refers to the real-time insertion of computer-generated content into a real scene (see [1], [2] for reviews). Typically, the observer's view of the world is via a head-mounted display (HMD) with a camera attached to the front. The video from this camera is modified and relayed to the HMD in real time. Early studies such as [4] superimposed two-dimensional textual information onto real-world objects. However, it has now become common to insert three-dimensional graphical objects. In this paper we extend these techniques by inserting captured images of live humans into augmented reality.

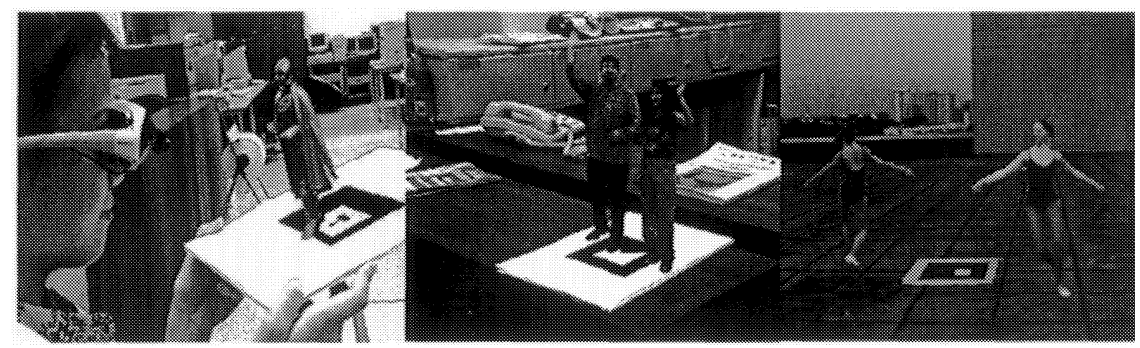

Figure 1. 3-D Live Augmented Reality. The user views the world via a head mounted display with a camera attached to the front (left). On each frame, the image of a live human actor is superimposed upon the card marker such that he/she appears to be a stable part of the three-dimensional scene. Multiple actors can be captured at once (centre), and display can potentially be at life size (right).

\section{ALGORITHM OVERVIEW}

In order to give the stable percept that the live content is threedimensional and present in the space with the observer we require that (i) the pose of the head-mounted camera relative to the scene is estimated, (ii) the equivalent view of the collaborator is generated and (iii) this view is rendered into the scene, possibly taking account of occlusions. The first of these problems is simplified by associating the virtual content with real twodimensional fiducial markers. We apply the method of Kato and Billinghurst [6] to estimate the Euclidean transformation between the head mounted camera and the marker. This transformation is used to define the 
position of a "virtual camera" viewing the live subject. Software for these procedures can be downloaded from [15]. The main technical problem is then to generate this virtual viewpoint at interactive speeds.

\subsection{Virtual Viewpoint Generation}

Novel view generation proceeds on a per-pixel basis as follows: First, we establish the 3-d position of the point on the object's surface that is projected to the virtual pixel. Second, we find the projected position of this point in nearby real camera images. Third, we estimate the colour of the virtual pixel by taking weighted sum of the colors in the real images. We consider each of these stages in turn.

We employ a shape-from-silhouette algorithm similar to that proposed by [10] in order to estimate the 3-d structure of the subject as seen from the virtual cameras view (see Figure 2). Fifteen cameras surround the subject. Each pixel in each camera is classified as either being part of the subject (silhouette) or the background. It can be shown that an approximation to the 3-d structure of the subject can be recovered from these silhouettes [8]. A ray is projected through each pixel in the desired image and an explicit search is carried out for where this first meets the object. In order to do this, the ray is projected back into the real cameras images, and the first intersection with the silhouette is sought. If no intersections are found the pixel is assumed to be background.

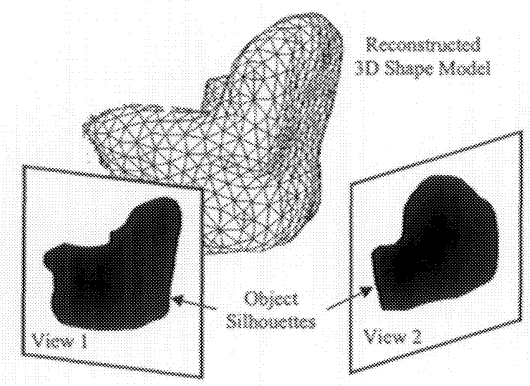

(a)

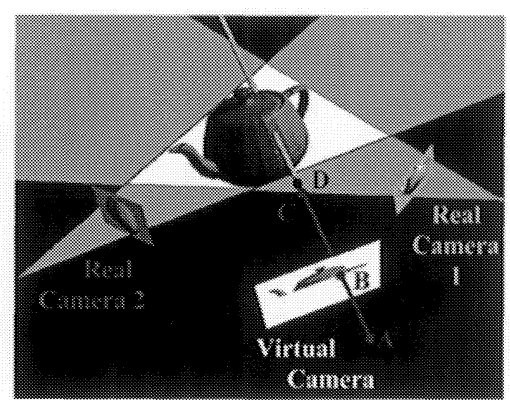

(b)

Figure 2 (a). Shape-from-silhouette principle - an approximation to the shape of a threedimensional object can be calculated based on its silhouette in a number of cameras views. This approximation is known as the visual hull [8]. (b) Image-based depth calculation. A line search is performed along a ray through each pixel in the desired view. This ray is projected into each of the real-cameras images and the first point at which it intersects the visual hull is established. Points from $\mathrm{A}$ to $\mathrm{C}$ have already been processed and fall outside the silhouette in both real images. Point $\mathrm{D}$ falls inside the silhouette in camera 1 but outside that of camera 2 and will similarly be rejected. 
If an intersection with the silhouette is established, we now have an estimate of the closest point along the ray that is on the surface of the subject. We now project this 3-D point back into the original camera views to establish the colour of the subject at that point. In general however, the position estimate is not exact, and the resulting depth errors cause the incorrect pixels to be chosen from each of the real camera views. In order to minimise the effects of these errors we sample only from cameras that are close to the virtual camera. For each of these cameras, we must check that the 3-d point is not occluded before allowing its colour value to contribute. Hence, we re-run the depth search algorithm on the pixel in the real camera. If the recovered depth lies sufficiently close in depth to the virtual pixel depth estimate, we allow the pixel colour to contribute. If not, then we assume that the point is occluded in this cameras view. A weighted sum of the colour estimates from each un-occluded real camera view is taken, where the weight is based on the relative proximity of the camera views.

The whole algorithm can be summarised in pseudocode as:

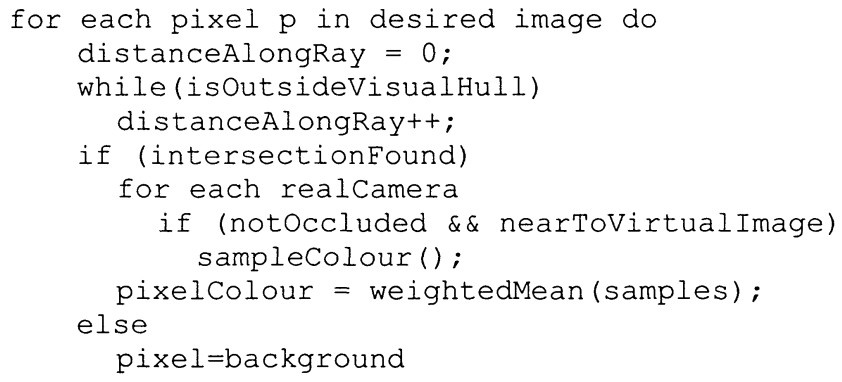

\section{$2.2 \quad$ System Details}

The overall system structure is depicted in Figure [3]. The scene was viewed through a Sony Glasstron HMD, with a front-mounted PremaCam SCM series color security camera which captured 25 images per second at a resolution of $640 \times 480$. For each frame, the Euclidean transformation relating the marker and camera co-ordinates was estimated and passed via ethernet to the rendering server. The rendering server returns the equivalent view of the subject, which is then drawn into the user's view in real time.

Fourteen Sony DCX-390 video cameras were equally spaced around the subject, and one viewed him/her from above. Five Dual $1 \mathrm{GHz}$ video-capture machines receive data from three cameras each. Each video-capture machine pre-processes the video frames and passes them to the rendering server via gigabit Ethernet links. This pre-processing consists of (i) classification of the pixel as foreground/ background, (ii) application of morphological 
operators (iii) compensation for geometrical lens distortion and (iv) lossless compression. The rendering server (a $1.7 \mathrm{GHz}$ Dual Pentium IV Xeon) takes all of these data and the transformation matrix and generates the novel view in each frame.

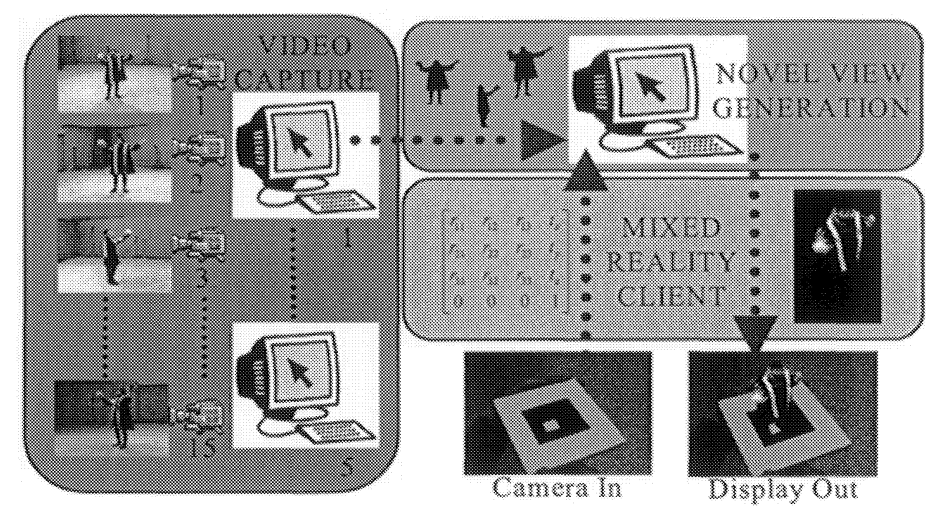

Figure 3. Live system overview - the subject is captured from all angles by 15 video cameras. For each frame, the augmented reality client establishes the Euclidean transformation relating the head-mounted camera and the marker and requests the equivalent "virtual view" of the subject. This view is computed from the 15 video streams and rendered back into the frame with a delay of $<100 \mathrm{~ms}$.

\subsection{System Performance}

The complexity of the algorithm is linear in the number of foreground pixels in the generated novel view, and substantially sub-linear in the number of cameras used. For typical live content, we can generate virtual views at $384 \times 288$ resolution at $25 \mathrm{~Hz}$ (frame rate of the head-mounted camera) with a latency of $<100 \mathrm{~ms}$. This allows interactive inspection and manipulation of the virtual content. The quality of the texture and the depth map produced depends on the proximity of the cameras surrounding the subject. As the cameras move closer, model quality improves but the space in which the subject can move becomes more restricted. For the figures in this paper, the captured space was $2.5 \mathrm{~m}$ high and $3.3 \mathrm{~m}$ in diameter with cameras that are 1.25 meters away. Assuming pixel accuracy in determining the silhouettes, this produces depth estimates which are within $\sim 0.5 \mathrm{~cm}$ of the true visual hull. 


\section{COMPARISON WITH PREVIOUS WORK}

We believe that this is the first system to present live captured 3-d content in augmented reality. However, there have been a number of previous attempts to generate live video avatars for virtual reality. One approach has been to use stereo vision to provide the depth information [11][12][13][14]. However, no current system of this type has positioned cameras 360 degrees around the subject. Moreover, stereo depth algorithms lack robustness resulting in flaws in the virtual view generation.

Two previous algorithms have used silhouette information to generate video avatars. Matusik et al. [10] present a similar image-based novel view generation algorithm. The principal differences are that (i) they generate the entire visual hull from the current camera angle, whereas we generate only the visible part. (ii) they bin the silhouette data and hence generate only an approximation to the visual hull whereas we reconstruct it exactly. Lok [9] has proposed a volumetric approach to shape from silhouette that makes ingenious use of hardware graphics capabilities. The advantage of this approach is that the resulting volumetric data allows simple interaction with virtual environments and can easily be viewed from multiple angles. The disadvantage is that computation scales linearly with the number of cameras and with the cube of rendering resolution.

The relative efficiency and scalability of our algorithm allows us to employ a 15 camera system. To the best of our knowledge, the closest comparable system uses only 5 cameras, and the model quality suffers accordingly.

\section{ENTERTAINMENT APPLICATIONS}

The ability to capture live action, and present it from an arbitrary angle has a great number of potential applications. These can broadly be divided into two areas: i) non-real time applications, in which the video data is buffered to disk and played back at a later date. Examples include captured content for video games or recorded three-dimensional concert performances. ii) real-time applications, in which the data is visualised as the subject is filmed. The canonical example of a real-time application for this system is visualisation of a collaborator in a virtual environment or computer game. In principle each player can view every other player from their own perspective throughout a game.

We have developed a novel entertainment application that utilises the "tangible" user interface created by associating virtual content with real world card markers (see Figure 4). The 3-D live system presented here 
allows us to visualise a player inside a virtual reality game-space. We view a miniaturised version of the virtual environment containing the player who is being tracked using standard VR technology. Following [7], we can use a card marker in the shape of a paddle to manipulate the position of the player in the environment. We can also use the paddle to interact with him in a natural way. We have developed a game in which we use the paddle to try and drop virtual objects onto the player's head. Objects are selected by moving the paddle close to them. We drop objects by tilting the paddle relative to the table. The player attempts to jump out of the way of the falling objects in real time. We implement a crude collision detection routing based on the depth information retrieved from the rendering algorithm.

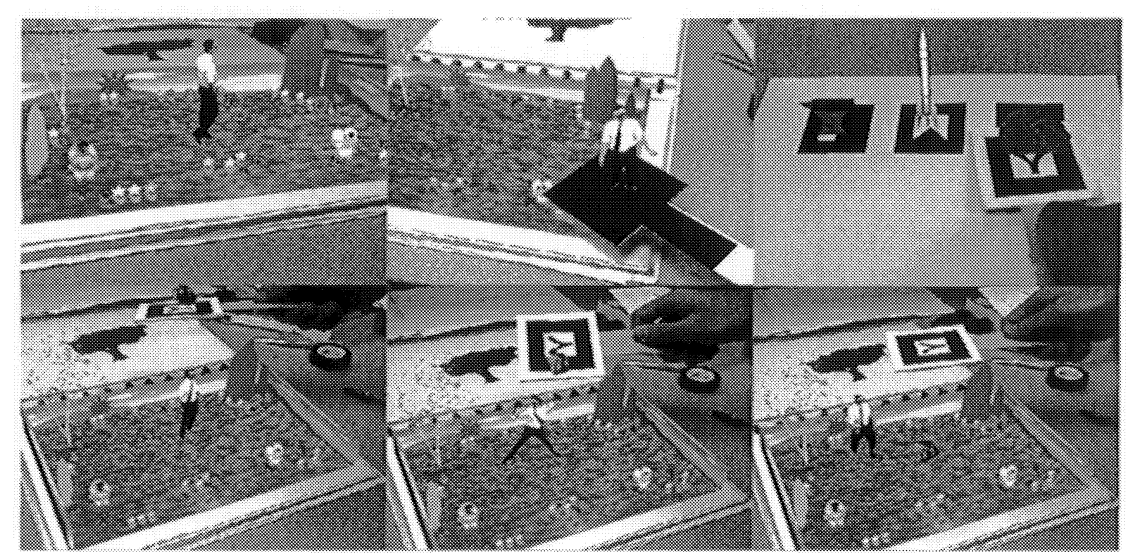

Figure 4. Example augmented reality entertainment application (sequence runs along each row in turn). Player 1 is immersed inside a virtual environment and is captured by the 3-D live system. Player 2 views a miniaturised version of the virtual environment superimposed upon the real world. Frame 1 shows how the 3-D live system allows him to see player 1 inside the environment. In frame 2, we show how player 2 can use tangible computing techniques to move player 1 about the environment with a card paddle. The next frame shows player 2 selecting a graphical object, which he then tries to drop onto playerl. Player 1 sees the object overhead in the environment and tries to evade it in real time.

\section{CONCLUSIONS}

We have presented a complete system for the capture and presentation of live content for entertainment applications. We generate arbitrary viewpoints of the subject at 25 frames per second with $<100 \mathrm{~ms}$ latency. This provides a tractable method for visualisation of collaborators in virtual or augmented reality. 


\section{REFERENCES}

[1] R. Azuma, "A survey of augmented reality," Presence, vol. 6, pp. 355-385, Aug. 1997.

[2] R. Azuma, Y. Baillot, R. Behringer, S. Feiner, S. Julier and B. MacIntyre, "Recent Advances in Augmented Reality," IEEE Computer Graphics and Applications, vol. 21, pp. 34-37, Nov./Dec. 2001.

[3] J. Cassell and K.R. Thorisson, "The power of a nod and a glance: Envelope vs. emotional feedback in animated conversational agents," Applied Artificial Intelligence, vol. 13, pp. 519-539, Jun. 1999.

[4] S. Feiner, B. MacIntyre, M. Haupt and E. Solomon, "Windows on the World: 2D Windows for 3D Augmented Reality," in Proc. UIST, 1993, pp. 145-155.

[5] T. Kanade, H. Kano, S. Kimura, A. Yoshida and O. Kazuo, "Development of a VideoRate Stereo Machine," in Proc. IRSC, 1995, pp. 95-100.

[6] H. Kato and M. Billinghurst. "Marker tracking and HMD calibration for a video based augmented reality conferencing system," in Proc. IWAR, 1999, pp. 85-94, 1999.

[7] H. Kato, M. Billinghurst, I. Poupyrev, K. Inamoto and K. Tachibana, "Virtual Object Manipulation on a table-top AR environment," in Proc. ISAR, 2000, pp. ????.

[8] A. Laurentini, "The Visual Hull Concept for Sillhouette Based Image Understanding," in IEEE PAMI, vol. 16, pp. 150-162, Feb. 1994.

[9] B. Lok, "Online model reconstruction for interactive virtual environments," in Proc. Symposium on Interactive 3D Graphics, 2001, pp. 69-72

[10] W. Matusik, C. Buehler, R. Raskar, S.J. Gortler and L. McMillan, "Image-Based Visual Hulls," in Proc. SIGGRAPH, 2000, pp. 369-374.

[11] J. Mulligan, V. Isler and K. Daniilidis, "Performance evaluation of stereo for telepresence," in Proc. ICCV, 2001, pp. 558-565.

[12] J. Mulligan and K. Daniilidis, "Real time trinocular stereo for tele-immersion," in Proc. ICIP, 2001, pp. 959-962.

[13] T. Ogi,, T. Yamada, K. Tamagawa, M. Kano and M. Hirose, "Immersive Telecommunication Using Stereo Video Avatar," in Proc. IEEE VR, 2001, pp. 45-51.

[14] R. Raskar, G. Welch, M. Cutts, A. Lake, L. Stesin and H. Fuchs, "The Office of the Future: A unified approach to image based modeling and spatially immersive displays," in Proc. SIGGRAPH, 1998, pp. 179-188.

[15] http://www.hitl.washington.edu/artoolkit/ 